\title{
DAS WERDEN UND DER INHALT DER IRANISCHEN VERFASSUNG*
}

\author{
Von MeHdi Tohidipur
}

\section{Das Werden der Verfassungsordnung und des parlamentarischen Systems im Iran}

Die iranische Verfassungsordnung ist entstanden als Institution der bürgerlichen Gesellschaft mit feudalen Resten.

Diese bürgerliche Gesellschaft war ihrerseits das Ergebnis der ökonomischen und sozialen Umwälzungen der Gesellschaftsstruktur im Iran und eine Folge der industriellen Revolution in Europa, an der der Iran nicht teilnahm und dadurch in ökonomische und technologische Abhängigkeit von europäischen Ländern geriet.

In der zweiten Hälfte des 19. Jahrhunderts, also in der Regierungszeit des Kadscharenkönigs Nasereddin-Schah, fand sich der Iran in den Kampf um die kolonialistische Vorherrschaft Europas in Asien verstrickt. Eine Europäisierung der kadscharischen Gesellschaft und des iranischen Marktes erfolgte unter dem ökonomischen und politischen Druck der damaligen beiden rivalisierenden Mächte England und Rußland. Diese Periode war „eine organische Fortsetzung der vorangehenden und eine unentbehrliche Voraussetzung für die Entwicklung des Irans im 20. Jahrhundert ${ }^{1 " .}$

Schon beim Ausbruch des ersten Weltkrieges war der Iran eine Brücke zwischen Rußland und der britischen Kronkolonie Indien. Diese Tatsache führte dazu, daß die Engländer den Süden und die Russen den Norden des Iran unter ihren politischen Einfluß nahmen. Diese politische Einflußnahme der beiden Mächte England und Rußland wurde dadurch intensiviert, verstärkt und auf die ökonomische Einflußnahme erweitert, daß die iranische Regierung, an ihrer Spitze der NasereddinSchah, des eigenen Vorteils wegen, zahlreiche und wichtige Konzessionen an diese beiden Mächte erteilte und den Handel mit dem Westen ständig erweiterte und so dem europäischen Raum weitere Einflußmöglichkeiten gab.

Direkte Handlesbeziehungen zwischen England und dem Iran wurden 1830 aufgenommen; vorher importierte der Iran englische Waren aus Indien. Der Seehandel über den "Persischen Golf" wuchs zwischen 1845 und 1865 um das Fünffache. Nach dem Abkommen von Turkmenchay zwischen dem Iran und Rußland, das nach den iranisch-russischen Kriegen zustande gekommen war und aufgrund dessen der Norden des Iran zwangsläufig russische Einflußsphäre wurde, erweiterte sich der Handel mit Rußland. Der russische Export nach dem Iran lag etwa bei einer Million Dukaten jährlich.

Im Kaspischen Meer wurden Schiffslinien, vorwiegend mit russischem Kapital, eröffnet. Im „Persischen Golf“ schuf man eine reguläre Dampflinie, die alle zwei Wochen Bombay und Basra verband und den iranischen Hafen Bandar Abbas anlief.

\footnotetext{
* Vgl. außer der im folgenden zitierten Literatur:

Browne, E. G.: $\quad$ The Persian Revolution of 1905-1909, Cambridge 1910

Ders.:

Press and Poetry in Modern Persia, Cambridge 1914

Bushehri, J.

Constitutional Law. Principles of Constitutional Law and Constitutional Law

of Iran, Teheran 1971

The strangling of Persia, London 1912
Iran zwischen den Weltmächten 1941-1948, Oberursel 1948

Morgan Shuster, W.:

Steppat, F.:

Wilber, D. N.:

Iran past and present, London 1948

1 Vgl. Jan Rypka: Iranische Literaturgeschichte, Leipzig 1959, S. 323.
} 
In den Jahren 1858/59 bauten Engländer im Auftrage der iranischen Regierung die erste Telegraphenlinie von Sultanieh nach Tabriz, die 1836 bis Julfa erweitert wurde.

Im Jahre 1870 legte die „Indo-European-Company“ eine Telegraphenlinie zwischen London und Teheran über Rußland.

1889 erhielt der Engländer Baron Julius Reute eine Bankkonzession auf sechzig Jahre mit dem Recht, sich im Lande mit jeglicher Art von Bankgeschäften, Erwerbszweigen und Finanz- und Handelsunternehmungen zu befassen. Am 2. September 1889 gründete der Konzessionär "The Imperial Bank of Persia“ mit einem Anfangskapital von vier Millionen Pfund Sterling.

Die Bank hatte das Monopol zur Herausgabe von Banknoten. Darüber hinaus war die Bank bevollmächtigt, in Einvernahme mit der iranischen Regierung Konzessionen zur Ausbeutung der Mineralreichtümer des Landes zu erteilen. Im gleichen Jahr, d. h. im Februar 1889, folgte die Erteilung einer Bahnkonzession an Rußland und kurz darauf eine Lotteriekonzession an England.

Nach seiner dritten Europareise ${ }^{1 a}$ und zur Deckung der Reisekosten erteilte Nasereddin-Schah am 8. März 1890 eine Tabakkonzession auf fünfzig Jahre an den Engländer Major G. F. Talbot. Talbot bekam so das Monopol, fünfzig Jahre lang Tabak innerhalb und außerhalb des Iran zu kaufen, zu verkaufen und herzustellen und mußte dafür 15000 Pfund Sterling jährlich sowie ein Viertel des Reingewinns an den Schah abgeben. Diese Konzession mußte rückgängig gemacht werden, da die Tabakpflanzer, -produzenten und -konsumenten empört waren und vereint mit den inzwischen erstarkten politischen und religiösen Gruppen und Bewegungen gegen die Erteilung der Tabakkonzession durch zahlreiche Kundgebungen, denen ein passiver Widerstand (Einstellung des Rauchens und Einkaufs der Tabakwaren bis zur Aufhebung der Konzession) folgte, protestieren.

Dies war die erste Protestbewegung, genährt durch die ansetzenden national- und politisch-liberalen und religiösen Bewegungen im Iran, die gegen die willkürlichen Handlungen des Schahs gerichtet waren und zum Erfolg führten.

Die geistige Wurzel der politisch-sozialen Bewegungen im Iran des 19. und 20. Jahrhunderts lagen in den Anfängen der kadscharischen Herrschaft.

Eine politisch-sozial relevante religiöse Bewegung im Iran, während der Herrschaft der Kadscharen, war die Babi-Bewegung, eine Bewegung, die antifeudalistischen und nationalliberalen Charakter besaß. 1844 erklärte sich ein junger Kaufmannssohn aus Schiras namens Ali Mohammad als Bab (= Tür) unter den islamisch-schiitischen Gläubigen und als der verborgene schiitische imam Mehdi, der am Ende der Zeiten wieder erscheinen soll.

Bab erklärte die schiitische Eschatologie symbolisch und meinte, man müsse unter dem „Ende der Zeiten“ das Ende einer Ära verstehen, und er selbst sei der Begründer einer neuen Ära, Vorläufer eines größeren „den Gott offenbaren wird“ und der Urheber einer neuen Zeit im religiösen und sozialen Leben und dies nicht nur im Iran, sondern in der ganzen Welt.

Beim Erfolg dieser Bewegung wären die Vorrechte des schiitischen Klerus beseitigt worden, und somit wäre ein positiver Einfluß auf das ethische Leben des Landes ausgeübt worden. Diese Bewegung wurde aber 1852 in grauenvoller Weise von der herrschenden kadscharischen Regierung ausgerottet. 1a vgl. Hans Leicht (Hrsg.): Ein Harem in Bismarcks Reich, das ergötzliche Reisetagebuch des Nasreddin-
Schah, Tübingen-Basel 1969. 
Nachdem die Babi-Bewegung niedergeschlagen wurde, suchte der sich neu herausgebildete iranische Mittelstand in den aus Europa kommenden liberalen Ideen das Mittel und die Möglichkeit, gegen die Autokratie des Schahs zu operieren und die Grundlagen des Despotismus zu vernichten.

In der Regierungszeit Nasereddin-Schahs erreichte der Kampf zwischen Tradition und Reform, Konservatismus und Modernismus seinen Höhepunkt. Unter den Vorkämpfern der intellektuellen Bewegungen, des politischen und religiösen Liberalismus sind zwei Persönlichkeiten zu nennen: Der nonkonformistisch-liberale Malkom Khan (1833-1908) und der Jamalluddin-al-Afghani (1838-1897), der für eine Wiedererweckung des Islam in liberaler und moderner Richtung kämpfte. Diese beiden Männer waren eher liberale Reformisten als radikale Revolutionäre. Vereint mit dem islamisch-schiitischen Klerus waren sie bemüht, den von Europa erfahrenen politischen Liberalismus im Iran zu verbreiten. Sie stellten die liberalistische Bewegung im Iran auf gleiche Stufe mit den parallelen europäischen Bewegungen einiger Jahrzehnte zuvor. In diesem Zusammenhang fehlte es ihnen aber an historischem und soziologischem Denken, denn der Liberalismus war kein Produkt der historischen Entwicklung der iranischen Gesellschaft, und er wurde durch diese Männer auf eine Grundlage mit feudal-ökonomischen Beziehungen gestellt.

Dies soll aber auf keinen Fall die entscheidende Rolle dieser großen Männer in der Reform- und Protestbewegung im Iran des 19. und 20. Jahrhunderts in Frage stellen. Auf der Basis der Aktivitäten dieser beiden Männer - vor allem durch Malkom Khans Zeitschrift "Quanun" und Afghanis Zeitschrift "Akhtar", die in London und Konstantinopel erschienen und als einzige Zeitung frei ihre Meinung über die Zustände im Iran äußern konnten - und aufgrund der sozialen und politischen Zustände kamen fortschreitende politische und revolutionäre Bewegungen aus allen Teilen des Landes auf.

Unter dem Einfluß von Jamalluddin-al-Afghani traten immer mehr Kleriker auf die politische Bühne. Die Macht und der starke Einfluß des Klerus führten fortwährend mit dazu, daß die lokalen Protestbewegungen, was sich im Falle der Tabakkonzession eindeutig zeigte, sich zu nationalem Protest und einer weitreichenden Bewegung ausdehnten.

Nasereddin-Schah versuchte durch die Einführung mancher kultureller und sozialer Neuerungen, wie z. B. die Gründung des ersten iranischen Polytechnikums, die Darul-Funun (1852), eine quasi weiße Revolution innerhalb seines despotischen Regierungssystems durchzuführen, um den aufkommenden sozialen und politischen Ansprüchen der liberalen Reformisten und revolutionären Freiheitskämpfern des Landes $\mathrm{zu}$ begegnen und damit die ansetzende bürgerliche Revolution in eine Evolution bzw. Reformbewegung bei Beibehaltung des bestehenden despotischen Systems zu transformieren.

Das Aufkommen der politisch und sozial bewußten Kräfte des Landes, latente und manifeste politisch-soziale Spannungen, die Konflikte und Interessengegensätze zwischen den Männern des Hofes und dem feudalen Adel und die Rivalität und Interessenkollision der beiden Mächte Rußland und England im Iran ließen aber die auf eigenem Vorteil basierenden und oberflächlich-reformistischen Bemühungen Nasereddin-Schahs ohne Erfolg bleiben. Er wurde im Jahre 1896 von einem Anhänger Jamaluddin-al-Afghanis ermordet, und „es war dies in der Geschichte Persiens ein einzig darstehender Fall: ein Herrscher fiel nicht einem ehrgeizigen 
Thronprätendenten oder dessen Clique zum Opfer, sondern wurde durch einen Revolutionär aus dem Volke ermordet. Und dieses Ereignis ist auch das erste sichtbare Glied der Kette von Veränderungen, durch die sich am Anfang unseres Jahrhunderts das Leben des modernen Iran zu formen beginnt ${ }^{2}$ “.

Der Großwesier Nasereddin-Schahs sorgte für einen ruhigen und ungestörten Thronwechsel.

Muzaffareddin, der als Kronprinz in Tabriz lebte, wurde nach Teheran berufen, und am 8. Juni 1896 bestieg er den Thron seines Vaters. So folgte dem energischen und selbstbewußten Nasereddin-Schah der außerordentlich fügige und dazu kranke Muzaffareddin-Schah. Der neue Herrscher „befand sich häufig außer Landes auf Reisen in Europa, und da er die leere Staatskasse für seine Reisen füllen mußte, begann mit ihm das zum Ruin führende System der Staatsanleihen, für die europäischen Länder Konzessionen auf Kosten der persischen Souveränität eingeräumt wurden. So gewährte im Jahre 1900 die russische Banque d'Escompte de Perse ein Darlehen von 2400000 Pfund Sterling gegen sämtliche Zolleinnahmen (außer der Provinz Fars und dem Persischen Golf). 1902 liehen die Russen wiederum 10000000 Rubel für die Erlaubnis, eine Straße Julfa-Teheran via Tabriz-

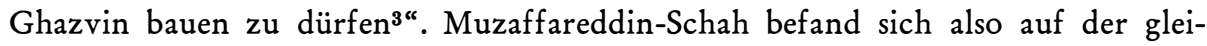
chen politischen Linie der Abhängigkeit von der finanziellen Hilfe der europäischen Mächte, Rußland und England, wie sein Vater.

Dieser Umstand führte u. a. dazu, daß die unter der Herrschaft NasereddinSchahs erstarkten revolutionären und liberalen Tendenzen während der Regierung Muzaffareddin-Schahs zum Ausbruch kamen. Die russische Revolution im Jahre 1905 gab den letzten Anstoß, vor allem vom Norden und Nordosten des Landes, der die revolutionären und liberalen Kräfte und mit ihnen, wenn auch mit ungleichen Grundgedanken, große Teile des islamisch-schiitischen Klerus zu entscheidendem Vorgehen veranlaßte und zur „Konstitutions-Bewegung“ führte. Die Forderung nach Verfassung und Nationalversammlung war nun nicht mehr zu umgehen.

Hier ist zu erwähnen, daß „obwohl vieles über den reaktionären Einfluß des Klerus zu sagen wäre, die Tatsache nicht zu leugnen ist, daß gerade Teile des Klerus in dieser Zeit die einzigen Rettungsanker - vielleicht noch mehr als die abstrakten bürgerlichen und philo-europäischen „Liberalen“ - für das Volk waren, das im Widerspruch $\mathrm{zu}$ allen Lehren des muselmanischen Rechts ausgesogen wurde" “. Muzaffareddin-Schah sah sich schließlich gezwungen, der Wahl einer „konstitutionellen Versammlung“, d. h. eines Parlaments (madjles-e schura-ye melli, „beratende Nationalversammlung “, und der Billigung einer Verfassung zuzustimmen.

Am 9. August 1906 wurde das Parlament feierlich eröffnet, und am 7. Oktober 1906 wurde in großer Eile mit der Ausarbeitung einer Verfassung, die wegen eben dieser Eile, des Fehlens einer überlieferten Verfassungsgrundlage und geringer verfassungsrechtlicher Erfahrung und Kenntnisse ihrer Verfasser relevante Unzulänglichkeiten im Inhalt und System aufweist, begonnen und schon zwei Monate später dem Muzaffareddin-Schah zur Ausfertigung und Verkündung vorgelegt. Am 30. Dezember unterzeichnete Muzaffareddin-Schah das Verfassungsdokument. Außer der Schaffung einer Verfassung bzw. einer konstitutionellen Monarchie hatte das Parlament eine Fülle von Aufgaben schnellstens zu erfüllen.

2 Vgl. Jan Rypka: a. a. O., S. 341.

3 Vgl. Alessandro Bausani: Die Perser, Stuttgart 1965, S. 165.

4 Vgl. Ebenda, S. 165. 
$\mathrm{Zu}$ diesen Aufgaben gehörten vor allem:

- Die Beseitigung der Finanznot des Landes, in die das Land durch den herrschenden Monarchen und seinen Vater geraten war.

- Die Schaffung der gesetzlichen Grundlagen für staatliche und administrative Tätigkeit.

- Die Entwicklung eines Reformprogramms zur Beseitigung oder Umgestaltung der alten und überlebten sozialen und politischen Ordnungen und Institutionen.

- Die Schaffung der gesetzlichen Grundlagen zur Umgestaltung der bisherigen Sozialordnung, insbesondere in bezug auf die feudal-herrschaftliche Ordnung.

- Die Herauslösung des Landes aus dem Machtbereich und dem Rivalitätsspiel der beiden Mächte Rußland und England.

Hinsichtlich der Finanznot ging die Regierung davon aus, daß man eine Anleihe von Rußland oder England oder von den beiden Mächten erhalten sollte. Das Parlament lehnte aber, nach heftigen Debatten, diesen Vorschlag ab und beschloß statt dessen, das Geld durch eine nationale Anleihe aufzubringen und durch eine Nationalbank verwalten zu lassen. Dieser erste entscheidende Beschluß des Parlaments wurde am 4. Dezember 1906 im ganzen Lande bekanntgegeben.

Wenige Tage nach der Unterzeichnung des Verfassungsdokuments, d. h. am 9. Januar 1907, starb Muzaffareddin-Schah. Sein Nachfolger wurde Kronprinz Mohammad-Ali, ein offener Gegner der Verfassung und des Parlamentarismus.

Mohammad-Ali-Schah war „vom ersten Augenblick an bemüht, die Rechte des Maglis (des Parlaments, M. T.) zu beschneiden. Dem Parlament gelingt es durch ein selbstbewußtes Verhalten und voll Unerschütterlichkeit, seine hauptsächlichsten Forderungen, trotz des Widerstandes des Hofes, durchzusetzen. Die erste derselben, die Errichtung der iranischen Nationalbank ... versetzt dem englischen und russischen Handelsprestige einen starken Schlag. Weiter fordert das Maglis die Entfernung der Ausländer aus der Staatsverwaltung und schließlich die Verminderung der königlichen Apanagen ${ }^{5 " .}$

Im Oktober 1907 mußte Mohammad-Ali-Schah, trotz seiner anfänglichen Weigerung, dem Druck des Parlaments nachgeben und das in der Nationalversammlung ausgearbeitete und beschlossene Ergänzungsgesetz zur Verfassung unterzeichnen.

Mohammad-Ali-Schah versuchte mit Unterstützung der beiden Mächte Rußland und England eine Wendung der Macht zu seinen Gunsten herbeizuführen, und dies tat er u. a. unter Verwendung aller möglichen Mittel des Terrors. Trotzdem gelang es ihm nicht, das Parlament auszuschalten. Schließlich ordnete er 1908 die Zerstörung des Parlamentsgebäudes an. Diese Maßnahme gab den Anstoß zum Ausbruch eines gewaltigen Aufstandes gegen den Mohammad-Ali-Schah, der vom einflußreichen islamisch-schiitischen Klerus, dem sich in der Kadscharen-Zeit neu herausgebildeten Bürgertum bzw. der städtischen Bürgerschaft und dem mächtigen Nomadenstamm der Bakhtiari unterstützt wurde. Und „so siegten nach einem fast zweijährigen Kampf doch die persischen Nationalisten. Dies war nur deshalb möglich, weil sie nicht bloß eine isolierte politische Gruppe waren, sondern weil wirklich das ganze Volk hinter ihnen stand. Wer nicht aktiv kämpfen konnte, unterstützte wenigstens durch warmes Interesse und flammende Begeisterung jeden Erfolg der nationalistischen Heere. Sogar in den Kaffeehäusern, wo die Sänger früher Bruchstücke aus dem Sah-name und aus den Klassikern rezitierten, wurden

5 Vgl. Jan Rypka: a. a. O., S. 343. 
in dieser Zeit die neuesten Nachrichten der revolutionären Presse vorgelesen und Verse zur Verherrlichung der Konstitution gesungen ${ }^{\text {" }}$.

Im Juli 1909 endete dieser Kampf zwischen dem Volk und dem Hof; die Aufständischen setzten Mohammad-Ali-Schah ab und erhoben seinen kaum zwölf Jahre alten Sohn Ahmad-Mirza auf den Thron. Ahmad-Schah eröffnete feierlich das Parlament, und der revolutionäre Nationalrat ernannte die Regierung.

Die neue Regierung ging energisch an die Arbeit und schuf Ordnung, insbesondere in den Finanzen, so daß deren Auswirkung sich schon im ersten Jahr ihrer Tätigkeit zeigte.

Kurz danach „machte sich in Europa jene Unruhe bemerkbar, die den ersten Weltkrieg ankündigte. Ihr Reflex dringt auch nach Iran. Ein für die Alliierten sowohl strategisch als auch durch seinen Mineralreichtum außerordentlich wichtiges Gebiet wird bald nach der Entfesselung des Krieges von Rußland und England besetzt. Das Elend ist größer als je zuvor "“.

Es begannen von neuem Revolten und Aufstände gegen die Okkupanten, und es herrschten wieder chaotische Zustände: Die Aufstände in Maschhad und einigen anderen Städten; Versuche Mohammad-Ali-Schahs zurückzuschlagen; Zersplitterung des Volkes in verschiedene politische Lager u. ä. Schließlich bereitete 1921 Reza-Khan als Befehlshaber der Kosakenbrigade einen. Staatsstreich vor, marschierte gegen Teheran und wurde Kriegsminister des neuen Kabinetts und der folgenden Regierungen.

Reza-Khan bemühte sich vor allem um die Verbesserung, Organisierung und Zentralisierung der iranischen Truppen und die Unterwerfung der teilweise unabhängigen Nomadenstämme. Im Februar 1924 übertrug das Parlament die Rechte eines obersten Kriegsherrn von Ahmad-Schah auf den, inzwischen zum Ministerpräsidenten gewählten, Reza-Khan.

Am 14. Februar 1925 übertrug das Parlament durch ein Gesetz die Stellung eines obersten Befehlshabers aller Truppen der nationalen Verteidigung und Sicherheit auf Reza-Khan, der inzwischen den Familiennamen Pahlavi angenommen hatte (aufgrund des Personenstandgesetzes von 1925), der dieses Amt in „aller Machtvollkommenheit und in den Grenzen der Staatsverfassung" auszuüben hatte.

Am 31. Oktober 1925 beschloß das Parlament, mit 80 von 85 Stimmen, AhmadSchah und mit ihm die Dynastie der Kadscharen abzusetzen und die vorläufige Regierung Reza-Kahn-Pahlavi zu übertragen und zur endgültigen Entscheidung eine neue Nationalversammlung einzuberufen. Am 12. Dezember des gleichen Jahres wählte das neue Parlament, mit 257 Stimmen bei drei Enthaltungen, RezaKhan-Pahlavi zum iranischen Kaiser (Schah-en Schahe Iran).

Seine Monarchie sollte, nach der Verfassungsänderung im Jahre 1925 und vor allem der Änderung der Artikel 36, 37, 38 der „Ergänzenden Verfassung8“, in der Weise erblich sein, daß sein ältester Sohn von einer Mutter iranischer Abstammung, der das 20. Lebensjahr vollendet hat, für regierungsfähig erklärt wird (Art. 37 u. 38 der „Ergänzenden Verfassung“ = EIV) und falls der König (Schah) keine männlichen Kinder haben sollte, wählt die beratende Nationalversammlung nach Vorschlag des Königs den Thronfolger (Art. 37 EIV). Erforderlichenfalls,

6 Vgl. Jan Rypka: a. a. O., S. 343.

7 Vgl. Ebenda, S. 344

8 Der Art. 36 legte die Monarchie in der Familie der Kadscharen fest; nach Art. 34 mußte die Mutter des Königs bzw. Thronfolgers eine Prinzessin sein; und Art. 38 erklärte den Thronfolger schon mit 18 Jahren für regierungsfähig. 
wenn der Thronfolger das gesetzlich bestimmte Alter noch nicht erreicht hat, sollte der König oder beratende Nationalversammlung einen Regenten bestimmen (Art. 38 EIV). Die heute geltende „Ergänzende Verfassung“ (Art. 38) bestimmt in diesem Falle die Kaiserin als Regentin, es sei denn, daß der Kaiser vor seinem Tode einen anderen Regenten bestimmt hat.

Die feierliche Eidesleistung des Reza-Schah-Pahlavi fand am 15. Dezember 1925 statt, und damit begann das Zeitalter der Pahlavi-Dynastie.

In der Regierungszeit Reza-Schahs, vor allem nach der Etablierung seiner Herrschaft kann man von einer De-facto-Ausschaltung der parlamentarischen Funktionen sprechen, denn in dieser Zeit war de facto die gesetzgebende und durchführende Gewalt Reza-Schah selbst. Das Parlament hatte in der Praxis nur noch formalen Charakter. Die Gesetzesvorlagen der Regierung wurden, fast ohne Ausnahme, ohne Gegenstimme verabschiedet, denn die Abgeordneten waren zwar formal vom Volke gewählt, in der Wirklichkeit aber Funktionäre des Regimes. Stimmte ein Abgeordneter gegen die Gesetzesvorlage der Regierung, so mußte er damit rechnen, daß er, abgesehen von eventuellen Repressalien, bei der nächsten Wahl nicht mehr aufgestellt wurde.

In seiner Regierungszeit ergriff Reza-Schah einige wichtige sozio-kulturelle Initiativen u. a.:

- Die Zentralisierung der Staatsgewalt, die durch permanente Unruhe im Lande und durch die zahlreichen Nomadenstämme bzw. -fürsten zersplittert war.

- Die Unterbindung der allzu großen Einmischung des islamisch-schiitischen Klerus in die politischen und kulturellen Angelegenheiten.

- Die Aufhebung der alten und Vergabe der neuen Wirtschaftskonzessionen.

- Die Regulierung und Verbesserung des ökonomischen Systems des Landes und der Staatsfinanzen, insbesondere durch Neuregelung des Steuersystems und des Ausgleichs zwischen Ein- und Ausfuhr.

- Die Verbesserung des Verkehrswesens durch Straßenbau und Einfuhr der Personen- und Güterbeförderungsmittel.

- Die Urbanisierung der Städte und den Anfangsversuch zur Industrialisierung des Landes.

- Die Entwicklung und Modernisierung des Bildungswesens.

- Die Gründung der Universität zu Teheran und der Akademie für iranische Sprache und Literatur (Farhangestane-Iran).

- Die Einführung eines Ehe- und Scheidungsgesetzes - bis zu dieser Zeit galt das islamische Recht.

Bei all diesen Maßnahmen blieben aber die durchgreifenden Reformen des Sozialgefüges aus.

Sie brachten keine radikale Umwälzung in der sozialen Klassenstruktur bezüglich der drei Hauptgruppen: die Oberklasse der Landbesitzer, die städtische Mittelklasse und die große Masse der Bauern und Arbeiter. Selbst das Landesreformprogramm von 1951 vermochte diese soziale Klassenstruktur nicht wesentlich zu verändern.

Da Reza-Schah „der Elite der großen Landbesitzer angehörte - die Tatsache, daß er einige von ihnen verfolgen ließ und ihr Land einzog, beweist nichts gegen das Gesagte, denn dazu waren auch sehr viel früher. Feudalherren fähig gewesen! 一, zog er vor, das unzufriedene Volk mit Schlagwörtern abzuspeisen, mit einem Nationalismus, der sich mehr auf das vorislamische Persien als auf die Tradition des 
islamischen Persiens bezog, das viel konkreter, auch historisch, mit der modernen persischen Kultur verbunden ist ${ }^{9}$ “.

$\mathrm{Zu}$ Beginn des $\mathrm{Zweiten}$ Weltkrieges versuchte der Iran strenge Neutralität zu bewahren. Diese neutrale Position konnte der Iran halten, solange sich die beiden Mächte Rußland und England als Gegner gegenüberstanden. Nachdem der Krieg zwischen Deutschland und Rußland erklärt war und sich die beiden Mächte England und Rußland in einem Beistandspakt zusammenschlossen, nutzte dem Iran die Neıtralitätserklärung nicht viel, denn im August 1941 marschierten die englischen und russischen Truppen im Iran ein und besetzten das Land.

Im September 1941 dankte Reza-Schah zugunsten seines Sohnes, des Thronfolgers Mohammad Reza Pahlavi ab und wurde von den alliierten Mächten exiliert und auf die Insel Mauritius geschickt.

Mit der Besetzung des Irans und der Abdankung Reza-Schahs wurden die politischen Gefangenen entlassen, und es trat eine Situation ein, die ähnlich war der Ära vor Reza-Schah mit zwei Ausnahmen:

- Nicht nur die Verstädterung, der Nationalismus und die Erziehung waren gewachsen, sondern die Gruppenaktivität umfaßte eine weite Skala von politischer Philosophie.

- Es ragten unter den politischen Trends vor allem die früheren sozialen Kräfte der linksradikalen, der liberal-konstitutionalen und der streng traditionalen bzw. religiös orientierten konstitutionalen Bewegung hervor. Obwohl von Reza-Schah und später von ausländischen Mächten unterdrückt, brachten sie es fertig, aufgrund der Kraft der früheren konstitutionalen Bewegung und ihrer moralisch und intellektuellen Autorität, zu überleben.

Die revolutionierende Periode (1891-1912) und die folgenden zwei Dekaden bezeugen den Ausbruch von Nationalgefühl auf vielen Gebieten. Die sozialen Bewegungen im Iran stimulierten das Wachstum von politischen Parteien und halfen, mit Hilfe der Presse, Dichtkunst und Prosa, ihr eigenes Gruppenbild zu gründen.

Obwohl diese sozialen Bewegungen die meiste Unterstützung von der bürgerlichen Mittelklasse bezogen, hatten sie einige Wirkung auf die Bauern und die Arbeiterklassen. Schon waren die unteren Klassen keineswegs gleichgültig ihrer eigenen Situation gegenüber. 1921 schlossen sich die Vereinigungen zusammen, um einen Zentralverband zu bilden, dessen Mitglieder 1925 auf 30000 geschätzt wurden. So errang die Arbeiterschaft in der Periode von 1906-1925 wesentliche Erfolge. Zum erstenmal erkannten beide Facharbeiter und ungelernte Arbeiter die Vorteile, sich $\mathrm{zu}$ einer Gewalt $\mathrm{zu}$ verbinden und teilzuhaben am allgemeinen Ziel und sie demonstrierten ihre Macht und Solidarität durch Teilnahme an Streiks und öffentlichen Demonstrationen. Erziehung zur politischen und sozialen Beteiligung wurde das Ziel aller sozialen und politischen Bewegungen.

Es ist wahr, daß soziale und politische Aktivitäten seit der Herrschaft Reza-Schahs gezügelt worden waren, und daß Reza-Schah versucht hat, eine absolute Monarchie wieder herzustellen, aber niemand wurde in dem Glauben irregeführt, daß „Autokratie“ eine neue Regierungsform für den Iran sei. Es gab zu viele Iraner, die sich noch erinnerten, welche Opfer ihre Väter für die konstitutionelle Regierung gebracht hatten. Die moralische und intellektuelle Autorität dieser Bewegung konnte nicht erschüttert werden.

9 Vgl. A. Bausani: a. a. O., S. 172. 
Die streng nationalistischen sozio-politischen Bewegungen, nach der Abdankung Reza-Schahs, opponierten gegen die Einmischung der westlichen Mächte in einheimische Belange und unterstützten die Aktivitäts-Programme der politischen Parteien zur Neugestaltung der politischen und sozialen Struktur der iranischen Gesellschaft.

Besser organisiert als der Rest, hatten die Linksradikalen bald ein Programm der Aktivität. Die linksradikale Hesbe Tudehe Iran (Iranische Massenpartei) begann mit der Entwicklung ihres Programms schon im September 1941, d. h. weniger als einen Monat, nachdem Reza-Schah abgedankt hatte. Die Ideologie und politische Philosophie der Konstitutionalisten fand ihren besten Ausdruck in Hesbe Iran (Iran Partei) gegründet im Mai 1944. Angegliedert waren die Hesbe Sahmatkeschane Melate Iran (Arbeitspartei des iranischen Volkes) gegründet 1952, und Niruye Sevom (Dritte Kraft) gegründet 1952. Die letztere Gruppe wurde der hauptsächliche Helfer von Mohammad Mosaddegh (Premier von 1951 bis 1953) und seiner Politik der Öl-Nationalisierung, und sie erhielten Unterstützung von den anderen Gruppen.

Traditionalismus lieferte die Basis für solch religiös orientierte Gruppen wie Fedaiyane Islam (Opfer für Islam) gegründet 1949, und Madjmae Modjahedine Islam (Verein der Kämpfer für Islam) gegründet 1951. Alle diese Gruppen, trotz doktrinärer Unterschiede, erstrebten die völlige Unabhängigkeit für den Iran, wie es in ihrer Unterstützung der OOl-Nationalisierung zum Ausdruck kam. Die Wiederbelebung dieser sozialen Bewegungen bestärkten in hohem Maße Presse und Massenmedien. Die Beziehung zwischen der Presse und den Parteien war aber reziprok, d. h. eine bestimmte Presse publizierte die Aktivitäten einer bestimmten Partei und umgekehrt unterstützten die Mitglieder dieser Partei das Blatt. Während der alliierten Besetzung des Iran (1941-1946) wurde die Presse eingeschränkt aber sie behielt doch noch ein gewisses Maß an Freiheit.

Während der Besetzung des Iran wurden (nach Schätzung) allein in Teheran über 100 Zeitungen und 25 Zeitschriften veröffentlicht. Auch die russischen und englischen Besatzungsmächte veröffentlichten mehrere Zeitungen, beide in ihren eigenen Sprachen und in Persisch. Die linksradikale Tudeh-Partei unterstützte zahlreiche Veröffentlichungen, unter ihnen Rahbar (Führer), Zafar (Sieg), Khaware Now (Neuer Osten), Rasti (Wahrheit). Im Verlauf des Jahres 1946 gab die Partei eine Zeitschrift unter dem Namen Mardom (Bevölkerung) heraus, die einmalig im Iran war bezüglich ihres inhaltlichen Formates, der Abwechselung ihrer Hauptinhalte und der Behandlung der Themen. Diese Zeitschrift präsentierte eine marxistische Analyse aller Lebensaspekte - des Sozialen, des Ókonomischen, des Künstlerischen und des Literarischen.

Die traditionelle soziale Macht, noch streng gebunden an religiöse Ordnung, machte von der Presse weniger als die Parteien Gebrauch; ihre Hauptveröffentlichungen waren A'ine Islam (Islamische Doktrin), Ruhe Asadi (Geist der Freiheit) und Nedaye Hagh (Stimme der Wahrheit).

Die Presse war nur ein Medium, das die politischen Gruppen benutzt haben, um sich Gruppenimage zu verschaffen. Ahnlich effektiv waren auch organisierte Gruppentreffen und Tätigkeiten, die auf die Vereini̧̧ung von Individuen mit gleichen Interessen, gleichem Alter und ausbildungsmäßigem Hintergrund zielten.

Während der vierziger Jahre hatte jede der wichtigen politischen Parteien im allgemeinen ihre eigene Jugendgruppe, Frauenorganisation, Turnverein, Diskus- 
sionsgemeinschaft und Arbeitervereinigung. Diese Gruppen trafen sich regelmäßig, um ihren Mitgliedern die Möglichkeit zu geben, Ideen und Meinungen miteinander auszutauschen. Diese Diskussionen erstreckten sich oft auf die Probleme der politischen Aktivität, des Arbeitsplatzes, der Schule, der Familie und vor allem auf die Analyse des aktuellen politischen Geschehens. Studenten trugen die Diskussionen der Gruppe in die Vorlesungsräume und versuchten die regulären wissenschaftlichen Diskussionen zu politisieren oder sie in politische Diskussionen zu transformieren. Diese Gruppen beschränkten sich nicht nur auf Aktivitäten und Diskussionen innerhalb ihrer Gruppen, sondern sie nahmen, den Forderungen ihrer zentralen Parteikomitees entsprechend, auch aktiv an öffentlichen Diskussionen und Demonstrationen teil.

Zum größten Teil wurden die Gruppentätigkeiten von den Linksradikalen bzw. der Tudeh-Partei, den Konstitutionalisten bzw. der Iran-Partei und den Traditionalisten bzw. den Feaiyane Islam dirigiert.

In neuerer Zeit wurde der Rundfunk ein mächtiges Medium der Information, Überzeugung und auch Manipulation.

Mohammad Mossadegh (Premier von 1951 bis 1953) demonstrierte sehr fähig, wie überzeugend Rundfunkansprachen politische Gefühle und Aktionen lenken. Wenn eine politische Krise entflammte, sprach er über eine nationale Schaltung und erhielt eine solide Unterstützung für sein Programm.

Mossadegh kam 1951 aufgrund der drohenden Merkmale einer latenten Unzufriedenheit und Widersprüchlichkeit in der schwierigen und schwankenden sozialen Lage an die Macht ${ }^{10}$. Mit seiner Politik der Ol-Nationalisierung und der inneren sozio-politischen Reformpolitik wurde er bald der westlichen Politik und dem Bestehen der Monarchie im Iran zu einer ernsten Gefahr. Diese Gefahr mußte beseitigt werden.

Mit dem Sturz Mossadeghs am 19. August 1953, mit Hilfe des amerikanischen Geheimdienstes (C.I.A.) und dem Verbot der oppositionell-progressiven Parteien endete die formal parlamentarisch-demokratische Herrschaftspraxis im Iran, und es begann die Zeit begrenzter sozialer und politischer Freiheit und der Wiederbelebung der Politik Reza-Schahs.

Der Parlamentarismus - im Rahmen der parlamentarisch-konstitutionellen Monarchie - blieb als "Norm" im System der Verfassung bestehen, die Herrschaftspraxis aber hörte auf, dessen Forderungen voll Rechnung zu tragen.

\section{Die Verfassung von 1906}

Die iranische Verfassung, wie sie am 30. Dezember 1906 durch MuzuffareddinSchah "dem Volk gewährt wurde" und am gleichen Tag in Kraft trat, ist in fünf Abschnitte unterteilt:

1. Die Bildung des Parlaments (Art. 1-14)

2. Zu den Pflichten, Kompetenzen und Rechten des Parlaments (Art. 15-31)

3. $\mathrm{Zu}$ der Überweisung von Angelegenheiten an die beratende Nationalversammlung (Art. 32-38)

10 Vgl. A. Bausani: a. a. O., S. 172. 
4. Anträge über die Angelegenheiten seitens des Parlaments (Art. 39-42)

5. Zu den Bestimmungen über die Bildung des Senats (Art. 43-51)

Eine Änderung des Textes der Verfassung von 1906 erging im Jahre 1949 (Änderung des Art. 48) und im Jahre 1957 (Änderung der Art. 4, 5, 6, 7) durch das „Konstituierende Parlament“ (=,Verfassungsgebende und -ändernde Versammlung“ aufgrund des „Zusatz-Artikels“ vom 8. Mai 1949).

Der erste Abschnitt beinhaltet die Regelungen über die Bildung der beratenden Nationalversammlung (Art. 1-11) - wobei die Texte der Art. 4, 5, 6, 7 im Jahre 1957 geändert wurden (Erhöhung der Zahl der Sitze in der beratenden Nationalversammlung von 162 auf 200 und der Dauer der Legislaturperiode von zwei auf vier Jahre usw.) -; die Bestimmung über die Ơffentlichkeit der Verhandlungen der Versammlung (Art. 13); die Regelung der Frage der Immunität der Abgeordneten (Art. 12) und die Festlegung, daß die beratende Nationalversammlung gemäß eines gesonderten Reglements (innere Geschäftsordnung) ihre internen Angelegenheiten regeln wird (Art. 14).

Der zweite Abschnitt enthält, obwohl er mit „Zu den Pflichten, Kompetenzen und Rechten des Parlaments" betitelt ist, ausschließlich die Rechte und Kompetenzen der Nationalversammlung und an wenigen Stellen auch die des Senats (Art. 15, 16, 17, 19).

So gehören zu den Kompetenzen des Parlaments: die Gesetzgebung (Art. 15, 16); die Aufsicht über die Durchführung von Gesetzen (Art. 27); die Regelung der Finanzangelegenheiten des Staates (Art. 18-20); die Zustimmung zur Veränderung der Staatsgrenzen (Art. 22) und zum Bau von Eisenbahnen und Verkehrswegen (Art. 26).

Außerdem werden, aus der politisch-historischen Erfahrung heraus, die Aufnahme von Staatsanleihen (Art. 25) und die Abschlüsse von Staatsverträgen und Abmachungen, Vergabe von Konzessionen (Monopolen) auf dem Gebiet des Handels, der Industrie und der Landwirtschaft, an Inländer und Ausländer (Art. 24), von der Zustimmung der beratenden Nationalversammlung abhängig gemacht. Im Art. 28 wird die Frage der Regierungsverantwortlichkeit vor dem Parlament nur unzureichend geregelt, die aber später in der „Ergänzenden Verfassung“ (Art. 60) zur ausreichenden Regelung kommt. Ebenfalls wird die Frage des Mißtrauensvotums des Parlaments dem Kabinett oder einem Minister gegenüber im Art. 29 unzulänglich geregelt, die später in der „Ergänzenden Verfassung“ (Art. 67) genauere Formulierung findet.

Der dritte Abschnitt beinhaltet Bestimmungen über das Petitionsrecht (Art. 32); die Unabhängigkeit der Abgeordneten in bezug auf die Stimmabgabe (Art. 38); die Vorlagen und Anträge der Regierung bzw. Regierungsmitglieder (Art. 33, 36, 37) und über die "geheimen Sitzungen“ im Parlament, die nicht nur unter Ausschluß der Ơffentlichkeit, sondern auch unter Ausschluß der von Parlamentspräsidenten für diese Sitzungen nicht bestimmten Abgeordneten stattfinden sollen (Art. 34, 35).

Der vierte Abschnitt enthält Bestimmungen über die Bedingungen der Annahme des Antrages eines Abgeordneten im allgemeinen (Art. 39) und wenn sich der Antrag auf einen verantwortlichen Minister bezieht (Art. 40, 41) und über die Auskunftspflicht der Minister der beratenden Nationalversammlung gegenüber (Art. 42). 
Der fünfte Abschnitt beschäftigt sich mit der Frage der künftigen Bildung einer zweiten Kammer (Art. 43), deren Mitglieder - insgesamt 60 - von „seiner kaiserlichen Majestät" und dem Volk je zur Hälfte gewählt werden sollen (Art. 45) und deren Geschäftsordnung der Zustimmung der beratenden Nationalversammlung bedarf (Art. 44). Nach der Bildung des Senats - dies geschah allerdings erst nach 1950, nachdem Anfang 1949 in der beratenden Nationalversammlung ein Gesetz zur Durchführung von Senatswahlen verabschiedet wurde - soll der Grundsatz der Gleichberechtigung beider Kammern in der Gesetzgebung gelten und die Beratung jeder Gesetzesvorlage und jedes Gesetzentwurfes nacheinander in beiden Kammern des Parlaments geschehen, ausgenommen sind hier die Finanzangelegenheiten des Staates, für welche die beratende Nationalversammlung allein zuständig ist (Art. 46, siehe auch Art. 18-20).

Die Schlußartikel enthalten Bestimmungen über die mögliche Auflösung und Wiedereröffnung einer der beiden Kammern des Parlaments (Art. 48, 49, 50) und die Beilegung der Streitigkeiten zwischen den beiden Häusern in bezug auf die Zustimmung zu einem Gesetzentwurf bzw. einer Gesetzesvorlage (Art. 48). Der Text des Art. 48 wurde im Jahre 1949 geändert, und zwar einseitig in Richtung auf Machtzuwachs des Monarchen, der demnach jederzeit die beiden Kammern, auch ohne Zustimmung des Parlaments, auflösen kann (die alte Fassung des Art. 48 schrieb die Zustimmung des Parlaments bei dessen Auflösung durch den Monarchen vor). Die iranische Verfassung von 1906 zeichnet sich bei aller Anlehnung an europäische Muster durch folgende eigenständige Merkmale aus: Sie entstand in einer soziopolitischen Situation, an welcher der Zeitdruck, bedingt durch die herrschende politische Instabilität und auch die Krankheit des dem Tode nahen MuzaffareddinSchah, einerseits und das Fehlen einer verfassungsrechtlichen und demokratischen Tradition und Grundlage andererseits, die Ausarbeitung einer Verfassung im Sinne des modernen juristischen Verfassungsbegriffs erschwerte.

Die iranische Verfassung von 1906 wurde nicht, wie in Demokratien westlicher Prägung, aufgrund bereits gegebener demokratischer Verhältnisse vom Parlament geschaffen. Sie hatte, wie übrigens auch deutsche Verfassungen, den Entwurf für die Baugesetze eines neuen Staatsgebildes erst zu liefern.

Die Verfassung von 1906 behauptet sich in Form einer Charta für das Parlament, denn sie regelt nur einen Teil des politischen Bereichs, nämlich den des Parlaments. Sie enthält keinerlei Aussagen über die Rechtsstellung der einzelnen, die Organe und Struktur des Staates, den Bereich ihrer Wirksamkeit und die Abgrenzung ihres Verhältnisses zueinander, und auch nicht über die Möglichkeit der Verfassungsänderung. Außerdem fehlt eine Präambel. Sie konzentriert sich fast ausschließlich auf die Organisations- und Funktionsbestimmungen für das Parlament. Die Verfassung von 1906 zeichnet sich außerdem durch die Betonung der Suprematie der Legislativversammlung; durch das Fehlen der Regelung des gegenseitigen Verhältnisses von Monarch und Volksvertretung, um Handlungen des Herrschers unter verfassungsrechtliche Kontrolle zu bringen; und durch das Fehlen von Bestimmungen über die Bildung und Funktion der Regierung.

Offensichtlich wollte man das Erreichte rasch in einer Charta niederlegen und somit die Grundlage für die Entwicklung einer „endgültigen“ Verfassung schaffen; zumal die Verfassung von 1906 eine Arbeit der Teheraner Parlamentarier war und nicht die der Vertreter des ganzen Volkes. 
Aber diese Entwicklung der Verfassung von 1906 wurde nicht vollzogen. Statt dessen - wohl aus der Überlegung heraus, daß der Akt der Verfassungsentwicklung, während der Herrschaft Mohammad-Schahs, dem engagierten Verfassungsfeind und offene Gegner des Parlamentarismus, zu einer absolutistischen Intervention seinerseits führen könnte - ging man daran, die systematischen und inhaltlichen Unzulänglichkeiten der Verfassung von 1906 durch die Schaffung einer „Ergänzenden Verfassung“ (1907) zu kompensieren.

\section{Die „Ergänzende Verfassung“ von 1907}

Wie bereits erwähnt, war die Verfassung von 1906 eine Arbeit der Teheraner Parlamentarier, die, basierend auf den Erfolgsergebnissen der Konstitutions-Bewegung und aufgrund der herrschenden politisch unstabilen Situation, in aller Eile die rechtlich gültigen und politisch wirksamen Fundamente eines parlamentarischen Systems zu legen versuchten. Als die Abgeordneten der anderen Provinzen in Teheran ankamen und mit ihrer parlamentarischen Arbeit begannen, gingen sie, mit ihren Teheraner Kollegen, energisch und mit voller Intensität daran, die Mängel des Verfassungswerks von 1906 sowohl in bezug auf den Inhalt als auch auf das System zu beseitigen bzw. zu kompensieren.

Die parlamentarische Arbeit der Mitglieder der beratenden Nationalversammlung war deshalb im Jahr 1907 zum großen Teil diesem Zweck gewidmet. Man führte aber, aus den bereits erwähnten politischen Gründen, keine Änderung und Verbesserung der Verfassung von 1906 durch, sondern bemühte sich um die Schaffung eines Ergänzungstextes zur geltenden Verfassung von 1906.

Diese 1907 entstandene „Ergänzende Verfassung“, die am 8. Oktober 1907 in Kraft trat, ist wie folgt gegliedert:

1. Allgemeines (Art. 1-7)

2. Die Rechte des iranischen Volkes (Art. 8-25)

3. Die Staatsgewalten (Art. 26-29)

4. Die Rechte der Mitglieder beider Kammern des Parlaments (Art. 30-34)

5. Die Rechte der iranischen Monarchie (Art. 35-57)

6. Über die Minister (Art. 58-70)

7. Die rechtsprechende Gewalt (Art. 71-89)

8. Über die Provinzial- und Unterprovinzialverbände (Art. 90-93)

9. Über das Steuerwesen (Art. 94-103)

10. Die Streitkräfte (Art. 104-107)

Eine Änderung des Textes der „Ergänzenden Verfassung“ von 1907 erging im Jahre 1925 (Änderung der Art. 36, 37) und im Jahre 1967 (Änderung der Art. 38, 41, 42) durch das „Konstituierende Parlament“ (= „Verfassungsgebende und -ändernde Versammlung" aufgrund des „Zusatz-Artikels“ vom 8. Mai 1949).

Im ersten Abschnitt der „Ergänzenden Verfassung“ wird die islamisch-schiitische Religion als Staatsreligion bestimmt (Art. 1), und die gesetzgeberische Tätigkeit des Parlaments auf der Basis des islamischen Rechts und der Vorschriften des Koran festgelegt, die durch ein Gremium, bestehend aus mindestens fünf Theologen, kontrolliert werden soll - Die religiöse Souveränität: „Das Adäquatprinzip“ - (Art. 2). Außerdem wird im ersten Abschnitt bestimmt, daß die eventuelle Veränderung der Grenzen des Landes, sowie seiner Provinzen und Unterprovinzen 
nur durch das Gesetz erfolgen kann (Art. 3); daß das Leben und Eigentum der im Iran lebenden Ausländer grundsätzlich geschützt ist (Art. 6); daß die offiziellen Farben der iranischen Fahne "grün-weiß-rot" sind, versehen mit dem Wappen des Löwen und der Sonne (Art. 5); daß Teheran die Hauptstadt des Reichs ist (Art. 4). Schließlich wird festgelegt, daß die in der Verfassung normierten Grundlagen des parlamentarischen Systems bzw. der konstitutionellen Monarchie weder teilweise noch ganz noch zeitweise außer Kraft gesetzt werden dürfen (Art. 7).

Der zweite Abschnitt beinhaltet die Grundrechte als Menschen- und Bürgerrechte. Garantiert wird: die Gleichheit der iranischen Bürger vor dem Gesetz - Gleichheitssatz - (Art. 8); der Schutz aller Personen in bezug auf Leben, Eigentum, Wohnung, Ehre (Art. 9); die Unverletzlichkeit der Wohnung (Art. 13); die Freizügigkeit der iranischen Bürger im ganzen Land (Art. 14); der Schutz des Eigentums (Art. 15, 16, 17); die Freiheit "des Lernens und Lehrens" von Wissenschaft, Kunst und Technik, unter Einhaltung der islamisch-religiösen Schranken (Art. 18); das Zensurverbot bei aller Art von Veröffentlichungen, deren Inhalt und Intention nicht im Widerspruch zu den Bestimmungen des Pressegesetzes und der islamischen Lehre stehen (Art. 20); die Versammlungsfreiheit, mit Ausnahme der unfriedlichen, bewaffneten und der Sicherheit und Ordnung des Landes gefährdenden Versammlungen (Art. 21); die Unverletzlichkeit des Brief-, Post- und Fernmeldegeheimnisses (Art. 22, 23).

Es wird weiter bestimmt, daß eine Tat nur aufgrund eines bestehenden Gesetzes bestraft werden kann - nulla poena sine lege - (Art. 12), daß die Freiheit der Person nur wegen eines begangenen Verbrechens oder Vergehens, oder aufgrund einer begründeten richterlichen Anordnung entzogen werden kann - Habeas Corpus-Regel - und die Gründe der Festnahme entweder sofort oder aber spätestens innerhalb von 24 Stunden nach der Festnahme dem Festgenommenen mitzuteilen sind (Art. 10); daß die Gründung von Schulen der ministeriellen Genehmigung bedarf (Art. 19); daß jeder Ausländer die iranische Staatsangehörigkeit annehmen kann und zwar nach den Bestimmungen eines besonderen Gesetzes - Staatsangehörigkeitsgesetz - (Art. 24).

Schließlich bestimmt die „Ergänzende Verfassung“, daß für Strafverfahren gegen öffentliche Beamte wegen Handlungen ihrer Verwaltung keine vorherige Ermächtigung notwendig ist mit Ausnahme der Bestimmungen über die Minister (Art. 25).

Im dritten Abschnitt werden die Prinzipien der Volkssouveränität - Alle Staatsgewalt geht von der Nation aus - (Art. 26) und der Gewaltenteilung - Es gibt drei stets voneinander getrennte Staatsgewalten: die gesetzgebende, die richterliche und religiös-schiedsrichterliche, die vollziehende Gewalt - (Art. 27, 28) dokumentiert. Außerdem wird ein Gesetz vorgesehen, wonach Provinzial-, Unterprovinzial- und Kreisverbände im ganzen Land einberufen und tätig werden sollen (Art. 29).

Der vierte Abschnitt bekundet, daß die Mitglieder der beratenden Nationalversammlung und des Senats die Repräsentanten des ganzen iranischen Volkes sind Grundsatz der Volksvertretung - (Art. 30); daß niemand gleichzeitig Mitglied beider Kammern des Parlaments sein kann (Art. 31); daß jeder der beiden Kammern des Parlaments das Recht zusteht, in den Angelegenheiten des Staates Untersuchungen und Nachforschungen anzustellen (Art. 33); daß ein Abgeordneter, der zu einem besoldeten, behördlichen Amt ernannt wird und es annimmt, seinen 
Sitz im Parlament sofort verliert - Inkompatibilität - (Art. 32); daß die Verhandlungen des Senats unverbindlich sind, solange die beratende Nationalversammlung nicht zusammengetreten ist (Art. 34).

Im fünften Abschnitt werden die Rechte und Funktionen der iranischen konstitutionellen Monarchie festgelegt.

Die Herrschaftsform des iranischen Staates ist die der konstitutionellen Erb-Monarchie (Art. 36).

Die konstitutionelle Erb-Monarchie ist im Auftrag des Volkes durch das „Konstituierende Parlament" der Person des Reza-Schah Pahlawi übertragen worden und wird in seinen männlichen Nachkommen von Generation zu Generation weiterbestehen (Art. 35, 36). Die Thronfolge fällt dem ältesten Sohn des Königs zu, der erst dann persönlich die Regierung übernehmen kann, wenn er sein 20. Lebensjahr vollendet hat (Art. 37, 38). Falls der König keinen Sohn hat, wählt die beratende Nationalversammlung auf Vorschlag des Königs den Thronfolger (Art. 37).

Der König kann ohne Zustimmung beider Kammern des Parlaments nicht gleichzeitig Oberhaupt eines anderen Staates sein (Art. 43). Folgende Kompetenzen werden dem König durch die „Ergänzende Verfassung“ eingeräumt:

Absetzung und Ernennung von Ministern (Art. 46); Verleihung von militärischen Dienstgraden, Orden und Auszeichnungen (Art. 47); Ernennung der leitenden Beamten der allgemeinen Verwaltung und des auswärtigen Dienstes mit Zustimmung des zuständigen Ministers (Art. 48); Bestätigung und Verkündung der Gesetze (Art. 49). Außerdem befehligt der König die Land- und Seestreitkräfte (Art. 50), erklärt Krieg, schließt Friedensverträge (Art. 51) und hat das Recht, die beiden Kammern des Parlaments zu außerordentlichen Sitzungen einzuberufen (Art. 54). Dabei ist die Person des Königs der Verantwortung enthoben; seine Minister sind dagegen in allen Fragen den Kammern gegenüber verantwortlich (Art. 44, 45).

Deshalb kann kein Akt des Königs - Gesetzesbestätigung und -verkündung, Erlasse - wirksam werden, wenn er nicht durch einen Minister, der damit allein die Verantwortung übernimmt, gegengezeichnet ist (Art. 45). Schließlich betont die Verfassung, daß der König keine anderen Befugnisse hat als die, welche ihm die Verfassung und die besonderen aufgrund der Verfassung erlassenen Gesetze ausdrücklich übertragen (Art. 57).

Der sechste Abschnitt enthält Bestimmungen über die vollziehende Gewalt bzw. die Aufgaben und Funktionen der Regierung.

Minister kann werden, wer Muslem, Iraner von Geburt, iranischer Staatsangehöriger und kein Mitglied der königlichen Familie ist (Art. 58, 59). Die Minister dürfen kein anderes Amt als ihr Ministeramt übernehmen (Art. 68). Sie sind vor den beiden Kammern des Parlaments verantwortlich - politische Verantwortlichkeit - und die Kammern können die Anwesenheit der Minister verlangen (Art. 60). Die Minister haben sowohl eine Ressort-Verantwortung als auch eine für das ganze Kabinett Kollektiv-Verantwortung (Art. 61). In keinem Fall kann eine mündliche oder schriftliche Anordnung des Königs einen Minister von seiner Verantwortlichkeit entbinden (Art. 64). Wenn die beratende Nationalversammlung und/oder der Senat, mit der absoluten Mehrheit ihrer Mitglieder, dem Kabinett oder einem Minister ihr Mißtrauen aussprechen, dann muß das Kabinett bzw. der betreffende Minister zurücktreten - Mißtrauensvotum - (Art. 67).

Die beiden Kammern des Parlaments haben das Recht, die Minister anzuklagen und sie vor den Revisionsgerichtshof zu stellen - rechtliche Verantwortlichkeit $=$ 
„impeachment“ (Art. 65, 69). Ein Gesetz wird die Fälle der „rechtlichen“ Verantwortlichkeit und die Strafen, die den Ministern aufzuerlegen sind, und die Art des Vorgehens gegen sie festlegen (Art. 66, 70).

Im siebenten Abschnitt werden die Funktionen und Grenzen der richterlichen Gewalt bestimmt.

Die richterliche Gewalt ist der ordentlichen Gerichtsbarkeit - für alle bürgerlichen Rechtsstreitigkeiten - und der religiös-geistlichen Schiedsgerichtsbarkeit - für religiös-rechtliche Streitigkeiten - anvertraut (Art. 71); sie wird durch die religiösen Schiedsgerichte und die ordentlichen Gerichte: - die Bezirksgerichte, die Kreisgerichte, die Provinzgerichte und den obersten Revisionsgerichtshof bzw. das „Oberste Staatsgericht" - ausgeübt (Art. 71, 73, 74, 75, 86, 88). Außerdem sieht die Verfassung die Bildung der Militärgerichte im ganzen Land vor (Art. 87). Die Richter und Gerichtspräsidenten werden aufgrund des Gerichtsgesetzes vom König ernannt (Art. 80). Sie können nur durch ein rechtskräftiges Urteil ihrer Ämter enthoben werden (Art. 81). Außerdem kann die Versetzung eines Richters nur mit seiner Zustimmung erfolgen (Art. 82). Ein Richter darf außer seinem Amt kein besoldetes Amt von der Behörde annehmen. Es sei denn, er ist gewillt, es ehrenamtlich auszuüben (Art. 85).

Der Staatsanwalt wird, mit Zustimmung des obersten geistlichen Schiedsrichters, vom König ernannt (Art. 83).

Die Verhandlungen sind öffentlich; Ausnahmen können bestehen bei den presserechtlichen, politisch-rechtlichen und sitten-rechtlichen Verhandlungen (Art. 77, 78, 79). Die Rechtsstreitigkeiten, welche die politischen Rechte betreffen, gehören zur Zuständigkeit der ordentlichen Gerichte (Art. 72).

Jedes Urteil muß begründet, mit den gesetzlichen Bestimmungen versehen und aufgrund des geltenden Rechts gefällt sein (Art. 78, 89).

Der achte Abschnitt enthält Bestimmungen über die Wahl der Mitglieder der Provinzial- und Unterprovinzialverbände (Art. 91), Funktionen und Befugnisse dieser Verbände (Art. 92) und die Öffentlichkeit des Haushalts und der Rechnungsberichte (Art. 93).

Der neunte Abschnitt beinhaltet die Grundbestimmungen über das Finanzwesen und verweist auf ein Gesetz, das die einzelnen Vorschriften über den Steuersatz, die Steuererhebung, die Steuerbefreiung und die Steuerermäßigung enthalten soll (Art. 94, 95, 96, 98, 99).

Außerdem sieht die Verfassung ein Gesetz zur Bildung und Organisierung eines Rechnungshofes vor (Art. 103). Die Mitglieder des Rechnungshofes werden durch die beratende Nationalversammlung für die durch das Gesetz festgelegte Frist ernannt (Art. 101). Der Rechnungshof ist mit der Prüfung und Abrechnung der Rechnungen der Finanzämter, der allgemeinen Verwaltung und aller Rechnungsbeamten gegenüber dem Staatsschatz beauftragt (Art. 102).

Im zehnten Abschnitt werden u. a. die Grundbestimmungen über die Rekrutierung und die Finanzierung der Streitkräfte aufgenommen. Die Art der Rekrutierung der armee, die Beförderung wie die Rechte und Pflichten der Soldaten werden durch Gesetz bestimmt (Art. 104). Den Soldaten können ihre Pensionen, Dienstgrade, Ehren nur aufgrund und im Sinne eines Gesetzes entzogen werden (Art. 107). Die Höhe der Verteidigungsausgaben wird jährlich durch die beratende Nationalversammlung beschlossen (Art. 105). Außerdem ist keiner fremden Truppe die 
Aufnahme in den Staatsdienst, Aufenthalt im Land oder Durchquerung des Staatsgebietes gestattet, es sei denn kraft eines Gesetzes (Art. 106).

Im folgenden seien die wesentlichen Charakteristika der „Ergänzenden Verfassung“ aufgezeigt.

Die „Ergänzende Verfassung" von 1907, die sich sehr stark an die Verfassung Belgiens vom Jahre 1831 anlehnt, spiegelt das politische Gewicht der Kräfte wider, die an ihrer Entstehung beteiligt waren: die liberalen Linken; die konservativliberale städtische Bürgerschaft; die konservative islamisch-schiitische Geistlichkeit.

Ihre Struktur und ihr normativer Gehalt stellen insoweit die Autobiographie eines Machtverhältnisses und einer Machtkonstellation in einer spezifischen, historischen und sozio-politischen Situation (Ansätze eines Übergangs von feudalistischer zu bürgerlicher Gesellschaftsformation) dar, die das klare Übergewicht der konservativ-liberalen und konservativen islamisch-schiitischen Kräfte aufzeigt.

Entgegen den Intentionen der Konstitutions-Bewegung, insbesondere der politisch Liberalen, einen liberal-demokratischen Rechtsstaat nach europäischem Vorbild zu schaffen, führte diese Bemühung, aufgrund fehlender historischer Basis, herrschender sozialer Klassenstruktur im Iran und der politischen OOkonomie der Verfassungen von 1906 und 1907, zur Abschaffung der absoluten Monarchie und Entstehung einer parlamentarisch-konstitutionellen Erb-Monarchie.

Es ist nicht verwunderlich, wenn die Verfassung von 1907 wie auch die von 1906 bis heute keine wesentlichen Änderungen erfahren hat, denn jene Machtkonstellation von 1906 und 1907 auf der Basis der damaligen sozialen Klassenstruktur, besteht im wesentlichen noch heute.

Seit 1907 hat im Grunde keine radikale Umwälzung in der sozialen Klassenstruktur bezüglich der drei Hauptgruppen: die Oberklasse Landbesitzer, die städtische Mittelklasse und die große Masse der Arbeiter und Bauern, stattgefunden. Die neuerlichen Veränderungen, die in der sozialen Klassenstruktur vorgenommen sind, tendieren danach, sich in den Ober- und Mittelklassen zu konzentrieren; die Stellung der Arbeiter und Bauern blieb aber unverändert. Insoweit manifestiert die Verfassung von 1907 noch heute die wirklichen Machtverhältnisse im Staat, die Verteilung des politischen Gewichts und Einflusses zwischen den verschiedenen Klassen, Schichten und Gruppierungen und schließlich die reale Stellung des Bürgers im Staat.

Auffällig ist die Hervorhebung der Rolle des Islams als Staatsreligion (Art. 1 EIV), als Normenkontroll-Institution im Parlament (Art. 2 EIV), als rechtsprechende Institution neben der ordentlichen Gerichtsbarkeit (Art. 71 EIV) usw. Abgesehen davon, daß diese Hervorhebung und der indirekte Einbau der islamisch-schiitischen Rechtsordnung in das Verfassungsrecht eine Diskriminierung der Minderheitsreligionen beinhaltet, steht sie im Widerspruch zum parlamentarischen System der Verfassung, insbesondere dort, wo die Verfassung die Bildung eines geistlichen Normenkontroll-Gremiums im Parlament vorschreibt. Denn das geistliche Gremium im Parlament übt (Art. 2 EIV) eine Beschränkungsfunktion der parlamentarischen Aktivität und Freiheit aus, und zwar dadurch, daß diesem Gremium durch die Verfassung ein absolutes und unveränderbares Vetorecht gegen alle Gesetzesvorlagen eingeräumt wird, falls nach seinem Ermessen eine Unvereinbarkeit mit dem islamisch-schiitischen Recht vorliegt. 
Nach dem Vorbild europäischer Verfassungsurkunden enthält auch die iranische „Ergänzende Verfassung" von 1907 einen Katalog von Grundrechten. Im wesentlichen lag der Aufzählung die Verfassung Belgiens von 1831 und die französische Verfassung von 1789 zugrunde. Es fehlen aber die Grundrechte der freien Meinungsäußerung, der Glaubens- und Gewissensfreiheit und die Regelung des Asylrechts. Eine Besonderheit ist der Einbau der islamisch-rechtlichen Schranken und Regelungen in das Grundrechtssystem (Art. 15, 18, 20, 21 EIV).

Alle Grundrechte unterliegen dem einfachen Gesetzesvorbehalt. Sie äußern keine den Staat bindende Wirkung, weil sie seinen Funktionen nicht vorgegeben oder übertragen sind, sondern lediglich vom Staat eingeräumte Rechtspositionen darstellen.

Inhalt und Auslegung der Grundrechte sind in das Ermessen der beratenden Nationalversammlung gestellt (Art. 27 EIV), die theoretisch durch eine einfache Mehrheit ihrer Mitglieder Grundrechtsbeschränkungen vornehmen kann. Dazu kommt, daß die Gerichte grundsätzlich die Verfassungsmäßigkeit der Parlamentsgesetze nicht überprüfen können. Die gerichtliche Kontrolle beschränkt sich auf eine Prüfung der Gesetzmäßigkeit. Der Bürger hat somit keine Kontrollinstanz, die er im Falle einer Grundrechtsverletzung anrufen könnte oder die jedenfalls die Anwendung der Verfassung überwachte.

In der „Ergänzenden Verfassung" von 1907 wurde die Volkssouveränität in den Rahmen einer parlamentarisch-konstitutionellen Erb-Monarchie eingeordnet.

Wenn auch die Idee des Ursprungs der Gewalt aus dem Volk dem Wesen des Islams nicht entsprach - da nach islamischem Staatsdenken die staatliche Gewalt aus der göttlichen Autorität fließt - , so drückte sich doch in der Repräsentation des Staates durch den Monarchen ein wesentliches Element der islamischen Theokratie aus.

Der König ist nach der Verfassung das Haupt und höchster Repräsentant des Staates mit sehr weiten Befugnissen sowohl im Rahmen der legislativen als auch der exekutiven Gewalt. Dabei ist er frei von jeder Verantwortlichkeit.

Nach der „Ergänzenden Verfassung" sollen die Vorschriften über den Aufbau und die Organisation der Rechtsprechung als traditioneller "dritter Gewalt" vorwiegend Gegenstand einfacher Gesetze sein. Sie haben deshalb nur zum Teil in die Verfassung Eingang gefunden. Dabei findet sich in der Verfassung kein Bekenntnis zur Rechtsstaatlichkeit, außerdem fehlen die grundsätzlichen Regelungen über die richterliche Unabhängigkeit, die Rechtsgarantien: Ne bis in idem und Ex-postfacto-Verbot, die Rechtsweggarantie, das Recht auf Verteidigung, der Anspruch auf rechtliches Gehör vor Gericht.

Die „Ergänzende Verfassung“ von 1907 enthält zwar grundsätzlich keine reinen Programmsätze, zeigt aber weithin eine in der inhaltlichen Weite und Unbestimmtheit der Regelungen begründete Offenheit. Sie stellt, neben wenigen kasuistischen Regelungen, doch sehr viele allgemeine Verhaltensmaxime (Richtlinien) auf. 


\title{
The Creation of an African Civil Service in Kenya
}

\author{
By VolKer VinNaI
}

Prior to independence the Kenyan civil service was primarily occupied with the collection of revenue to make the colony selfsupporting, with the maintenance of law and order and the creation of favourable conditions for the White agricultural sector and its supporting infrastructure. In Kenya Europeans filled the top posts in the service, the middle level was occupied by Asians, whereas Africans were recruited only for the jobs at the bottom. Even thoug they represented 80 per cent of the entire service in 1960 Africans occupied few of the better paid jobs.

With the approach of independence the colonial government started a rapid Africanization programme which transformed the service from a colonial one with limited functions into a national one geared to the economic and social development of the country and its African population. Shortly after independence virtually the entire administrative cadre of the service was filled with Kenya citizens, mostly Africans.

This speedy Africanization led to three long term consequences. First, the forcible retirement of British civil servants created a large financial commitment for the new state, which was finally terminated in 1972 when the British government took over the payment of pensions for its citizens. Second, Africanization took place within the old salary structure even though its upper scales were geared to attract Europeans for service overseas. Finally this rapid Africanization blocked the avenues of advancement for those who joined later even though they are better educated than their superiors.

Localization of the civil service has so far meant largely the Africanization of posts - the replacement of Europeans and Asians by Africans. Virtually no attempt has been made to change the practices and procedures of the administrative system. Africanization has in no way altered the structure of the service but only its personnel.

Kenyanization in the sense of re-shaping of institutions to serve local conditions and demands as distinct from replacing expatriates is now at least being recognized. With the Africanization of personnel almost completed, those other aspects of localization will perhaps be identified and remedied.

\section{Origin and Substance of the Iranian Constitution}

\section{By Mehdi Tohidipur}

We have seen that the Iranian Constitution, which came into effect immediately after the end of the Constitutional Movement (1906), was drafted in the euphoric atmosphere of victory and represented an establishment of the middle class with feudal remnants. It reflects the political importance of the groups which participated in its founding, i. e. the liberal Left, the conservative-liberal urban middle class, and the conservative islamic-shiit clergy. The form and normative substance of the Iranian Constitution therefore may be regarded as the autobiography of a political and economic balance of power in a specific historical and 
socio-political situation (beginnings of a change from a feudal system towards a middle class society).

The predominance of the conservative-liberal, especially the conservative islamicshiit group is evident; it explains why the change from a feudal towards a middle class society could not fully succeed. Mainly as a consequence of the predominating conservative groups, the plan of the Constitutional Movement, especially the liberal and revolutionary groups to create a liberal democracy based on the rule of law ended in a compromise; a representative, constitutional monarchy through inheritance was established. Thus the ideas of the Constitutional Movement have not all been expressed in the written Iranian constitution, especially in the amendments to the constitution of 1907.

When drafting the constitution, the conservatives did not completely eliminate the revolutionary ideology that had grown out of the revolutionary movements until 1906; it was, however, largely transformed into a conservative-reformist factor. This explains why the conservatives considered the Belgian Constitution of 1831 and some parts of the French Constitution of 1791, which provided for a representative, constitutional monarchy through inheritance, to be a more appropriate practical model than the 1793 Constitution of the French Republic or that of the Third Republic of 1875 which contained the provisions for a purely republican system. 